\title{
The Synergy Tool: Making Important Quality Gains within One Healthcare Organization
}

\author{
Enoch Ho ${ }^{1, *}$ (D), Elaine Principi ${ }^{1}$, Charissa P. Cordon ${ }^{1, *}$, Yayra Amenudzie ${ }^{1}$, Krista Kotwa ${ }^{1}$, \\ Sarah Holt ${ }^{1}$ and Maura MacPhee ${ }^{2}$ \\ 1 Quality and Performance, Hamilton Health Sciences, Hamilton, ON L8V 1C3, Canada; \\ principi@hhsc.ca (E.P.); amenudzie@hhsc.ca (Y.A.); kotwa@hhsc.ca (K.K.); holtsar@hhsc.ca (S.H.) \\ 2 School of Nursing, University of British Columbia, Vancouver, BC V6T 2B5, Canada; \\ maura.macphee@nursing.ubc.ca \\ * Correspondence: hoenoch@HHSC.CA (E.H.); cordonc@HHSC.CA (C.C.)
}

Received: 26 July 2017; Accepted: 3 September 2017; Published: 7 September 2017

\begin{abstract}
Background: Evidence-based clinical care delivery begins with comprehensive assessments of patients' priority needs. A Canadian health sciences corporation conducted a quality improvement initiative to enhance clinical care delivery, beginning with one acute care site. A real-time staffing tool, the synergy tool, was used by direct care providers and leadership to design and implement patient-centered care delivery. The synergy tool is the patient characteristics component of the Synergy Model $^{\mathrm{TM}}$, developed by an expert panel of nurses in the 1990s. Since then, the tool has been effectively used to assess a variety of patient populations on eight important characteristics, informing real-time staffing decisions. Methods: Plan-Do-Study Act cycles were managed by department-based project teams with assistance from business analytics and a quality/safety officer. Results: Initial findings demonstrate reductions in nurse missed breaks, improved workload management, and significant increases in staff engagement. Conclusions: The synergy tool is an easy-to-use tool that can be used to highlight priority care needs for individual patients or specific patient populations. The tool informs real-time staffing decisions, ensuring a better fit between patient needs and nurse staffing assignments. Although this initiative began with nurses, project work is expanding to include inter-professional teams.
\end{abstract}

Keywords: synergy tool; patient-centred care; PDSA cycles; quality improvement collaboratives

\section{Introduction}

Of critical importance to healthcare institutions is evidence-based clinical care delivery. Hamilton Health Sciences Corporation (HHSC) embarked on a quality improvement (QI) journey to design and implement a new care delivery model that would improve the quality and safety of patient care management, and engage and empower direct care nursing staff. Collaboration among direct care nursing staff, quality specialists, inter-professional team members (e.g., medicine, pharmacy, social services), and operational leadership supported a process based on the Plan-Do-Study Act (PDSA) framework; the PDSA framework serves as a backdrop for this article.

HHSC has seven care sites including acute care-tertiary, specialized children's care, regional rehabilitation, regional oncology, community hospital, urgent care, complex continuing care, palliative care, trauma/emergency services, and ambulatory care. To date, the work outlined is being undertaken within the acute care-tertiary care site. Our initial pilot on a hematology unit has since spread to a surgical oncology inpatient unit and a medical Alternative Level of Care (Medical-ALC) unit that supports seniors awaiting transition to home or residential care. Plans for continued spread will be discussed later in this paper. 


\section{When Did We Begin Our Journey?}

The trigger for this QI work stemmed from leadership and staff concerns related to declining morale, recruitment and retention problems, and quality and safety issues on the inpatient hematology unit. There were inconsistencies in how assignments for nursing staff were made from one day to the next, which caused friction among team members, complaints about workload, and potential and actual quality/safety risks to patients and staff. These challenges were complicated by increasing patient acuity and varying levels of nurses' skill and knowledge.

A designated quality/safety project team conducted a literature review on nursing care delivery models, locating literature on the American Association of Critical Care Nurses' Synergy Model ${ }^{\mathrm{TM}}$ (Curley 2007). This model consists of two components-a patient characteristics component and a nurse competencies component. The nurse competencies component of the model was originally developed for registered nurses (RNs) only. The patient characteristics component of the model can be used on its own in healthcare settings where the skill mix includes RNs and registered practical nurses (RPNs), unregulated care aides, and allied health professionals (e.g., respiratory therapists). The Synergy Model ${ }^{\mathrm{TM}}$ originated in United States (US) critical care settings, but its use expanded to different healthcare contexts, such as pediatric care (Gralton and Brett 2012), cardiac intensive care (Khalifehzadeh et al. 2012), and medicine-surgery (Carter and Burnette 2011).

The patient characteristics component is operationalized as a patient needs assessment tool known as the 'synergy tool.' In the tool there are eight patient characteristics, and each characteristic is scored from 1 (high needs) to 5 (low needs). Nurses and their management use the synergy tool to quickly identify patient priority needs based on the following eight characteristics: complexity, resiliency, vulnerability, stability, predictability, capacity to participate in own care, capacity to make own decisions, and access to resources. Table 1 contains the eight characteristics and their operational definitions. Once patient priority needs are identified, the numbers and types of nurses are more accurately determined, creating a 'synergy' between patient needs and nurse competencies. In settings with skill mix (e.g., RNs, RPNs) staffing decisions are based on legal scopes of practice and experience with the patient population and setting.

Table 1. The Eight Synergy Tool Patient Characteristics.

\begin{tabular}{|c|c|}
\hline Characteristic & Operational Definition \\
\hline Stability & $\begin{array}{l}\text { The ability to maintain a steady state. Stability can be used to describe physiological, } \\
\text { psychological, emotional, and family or social stability. }\end{array}$ \\
\hline Complexity & The intricate interplay of two or more systems, including body, family, and social systems. \\
\hline Predictability & $\begin{array}{l}\text { To expect a certain trajectory of illness. While most patients have a predictable course of } \\
\text { illness, some individuals do not respond in the typical fashion. }\end{array}$ \\
\hline Resiliency & The capacity to return to baseline functioning. \\
\hline Vulnerability & Susceptibility to stressors that may adversely affect outcomes: risk of harm to self or others. \\
\hline $\begin{array}{l}\text { Participation in } \\
\text { Decision-making }\end{array}$ & The extent to which the patient and/or family can engage in making informed decisions. \\
\hline Participation in Care & The extent to which the patient and/or family participate in care activities. \\
\hline Resource Availability & $\begin{array}{l}\text { Patient, family, or community resources to support patient care and well-being (e.g., } \\
\text { physiological, social, technical, financial resources). }\end{array}$ \\
\hline
\end{tabular}

In one study that examined the psychometric properties of the synergy tool, an exploratory factor analysis yielded a 2 -factor solution explaining about $70 \%$ of the variance. Five items (vulnerability, stability, complexity, resilience, and predictability) loaded on the first factor. Three items (participation in decision-making, participation in care, resource availability) loaded on the second factor. With respect to discriminant validity, for an expert sample of nurses and samples of direct care nurses, synergy tool item ratings varied by population, as expected. Means scores for critical care patients, for example, were statistically different for each of the eight characteristics (e.g., more complex, more unpredictable, fewer personal resources) than for patients on general medical-surgical units. Overall, this study concluded that the patient characteristics component of the Synergy Model ${ }^{\mathrm{TM}}$, 
the synergy tool, is a valid and reliable assessment tool that can be used for different patient populations and employed by nurses with varying levels of clinical expertise (Brewer et al. 2007).

In British Columbia, Canada, year-long pilots were conducted with the synergy tool across eight volunteer sites, consisting of two sites for each of the following sectors; acute care, community/public health, mental health and residential care settings (MacPhee et al. 2011). These pilots were supported through a collaboration between the provincial nurses' union, health regions' chief nursing officers, the provincial Ministry of Health, and academic nurse researchers. The over-arching purpose for this collaboration was to address nursing workload issues, particularly staffing. The synergy tool was used by direct care nurses and their managers to assess patients' priority care needs and collaboratively determine staffing guidelines (e.g., staffing levels or nurse: patient ratios, skill mix). Because these pilot projects were one year in length, only qualitative evaluations were done at the eight sites. Overall, [the tool] "helped clarify who was best suited to provide care for patients with particular needs; the model's patient-centered philosophy enhanced collaboration and teamwork around the common goal of better care provision, and the model and tool provided staff with a unified language, a systematic scoring approach and objective staffing guidelines" (MacPhee et al. 2011, p. 52). The synergy tool has also been introduced to acute care sites in Saskatchewan, Canada (Rozdilsky and Alecxe 2012), and this report reflects our implementation of the tool in Ontario.

\section{Materials and Methods}

Some evidence exists to support the positive impact of QI collaboratives on the quality of healthcare delivery. Collaboratives consist of multi-disciplinary team members from different organizational departments, working together to accelerate improvement (Schouten et al. 2008). At HHSC, a QI collaborative, including leaders from clinical programs, professional practice, data analysts, and direct care health care professionals formed the core group that implemented the synergy tool project within each clinical program. The core group worked collaboratively at all phases of the project, including the introduction and adaptation of the synergy tool for each patient population; evaluation metrics, including staff surveys and human resources data; and the development of patient scoring and staffing guidelines.

The Plan-Do-Study-Act (PDSA) QI approach used at HHSC is an adaptation of the Institute for Healthcare Improvement's Model for Improvement. It is a cyclical process and includes four steps to guide the continuous improvement process (Berwick 1996). The cycle begins with the Plan step, which includes problem identification, goal setting, defining process and outcome indicators, and developing a plan to address the problem. The HHSC Plan step focused on synergy tool validation for the inpatient hematology unit. A project lead who is an expert hematology nurse was appointed to oversee synergy tool adaptation for the hematology unit. Subsequently, this nurse also supported the implementation of the synergy tool in other areas at HHSC.

The second step is the Do step, which entails implementation of the plan. In the Do step, we utilized a "lunch and learn" method to introduce the synergy tool to the staff (Mawhinney 2010; Straka et al. 2013). We also used lunch and learn sessions during the Do steps of subsequent monthly PDSA cycles to garner feedback from direct care nurses as we made adaptations to synergy-based patient assessment guidelines and nurse staffing guidelines. Staff engagement through this approach was highly successful — 'smoothing' the introduction of the tool into normal unit operations. During the Do phase, other staff communications about the synergy tool took place during daily safety huddles and during monthly staff meetings.

During the third Study step, process and outcome indicators were monitored, and an analysis of further areas for improvement was done (Mainz 2003). During this step, the HHSC quality/safety specialist on the project team worked closely with nursing leadership to collect baseline data on nurse utilization and patient outcomes. These data were reported back to the QI collaborative team at weekly meetings. During the Act step, goals, methods, and indicators were adjusted accordingly. An example of one process indicator, for example, was synergy tool training effectiveness. To ensure 
staff inter-rater reliability / agreement of at least $90 \%$ (outcome indicator), periodic spot checks were conducted by synergy tool super users and direct care nurses' synergy scores for their assigned patients. Subsequent PDSA cycles were used to integrate use of the synergy tool within other units, including the Medicine/Alternate Level of Care unit and a Surgery unit. These units were volunteer units who had leadership support and an expert nurse project lead and/or educators available to introduce and continuously evaluate uptake and success of the synergy tool via PDSA cycles.

Appendix A provides an example of how we used PDSA cycles in this project.

\section{Results}

Using the synergy tool, we were able to learn about the patient population profile by site, staff perceptions on the manageability of their workload, staff engagement, factors that contribute to workload, and the skills sets of nurses on the unit. By using these data in continuous PDSA cycles, we were able to devise a timely, efficient, and systematic process for using the synergy tool in real time decision making, which includes matching patient care needs with the appropriate nurse.

\subsection{Spider Charts of Typical Patient Profiles by Site}

The patients' mean monthly synergy scores for each patient characteristic were used to create a graphic display of specific unit's patient profiles. Spider charts are a graphic display of three or more variables, and each spoke on the web represents a different variable (Chambers et al. 1983). As shown in Figure 1, the burden or intensity of care for a typical inpatient medical patient is mostly determined by stability and predictability characteristics; while Figure 2 shows that for surgical oncology patients, the highest burden/intensity is most closely determined by the complexity and predictability of underlying medical conditions and the capability of patients to participate in post-operative care regimens. In Figure 3, among the Medical/Alternative Level of Care patients, who are primarily frail seniors, resilience is the highest burden/intensity synergy score. Note: At HHSC, each patient characteristic is scored on a scale from 0 (least burden/intensity) to 5 (highest burden/intensity).

\section{Medical}

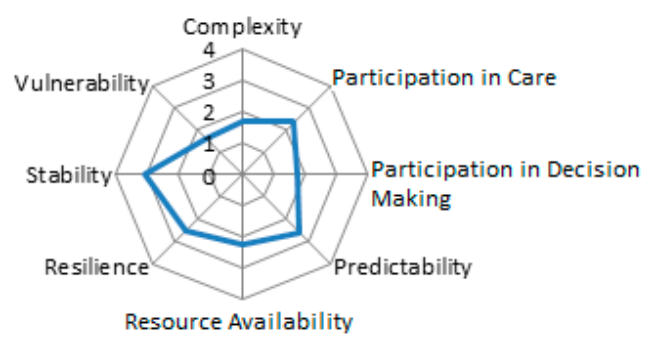

Figure 1. Spider Chart of Mean Synergy Scores for Patients on the Hamilton Health Sciences Corporation (HHSC) Medical Unit.

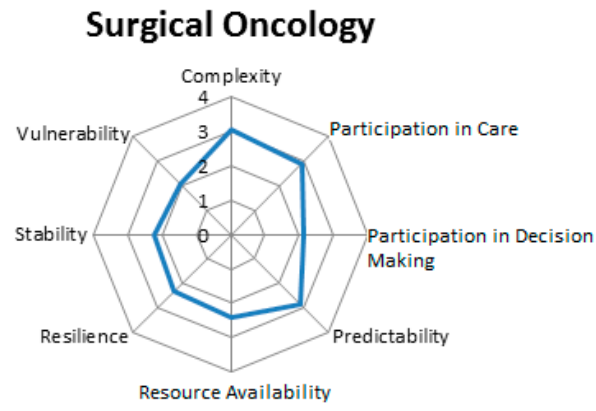

Figure 2. Spider Chart of Mean Synergy Scores for Patients on the HHSC Surgical Oncology Unit. 


\section{Alternate Level of Care}

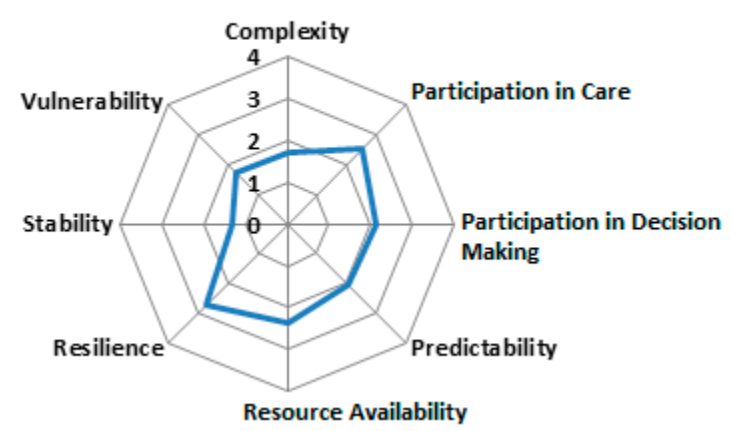

Figure 3. Spider Chart of Mean Synergy Scores for Patients on the HHSC Alternate Care Level Unit.

\subsection{Nurse Perceptions of Workload Management}

Other data gathered during PDSA Study steps focused on nurse perceptions of workload manageability. We created an easy survey that nurses were able to complete on a weekly basis after synergy tool implementation. This survey was distributed by the synergy unit champions and completed anonymously by nurses. Although this was a crude determination of workload management, the responses guided project team discussions and prompted constructive, testable QI changes via PDSA cycles. As shown in Table 2, we were able to review fluctuations on a weekly basis and explore the reasons for fluctuations and the factors that contributed to the unit's busyness. Over time, we have witnessed a more even distribution of nurses' workloads.

Table 2. Workload Survey.

\begin{tabular}{lllll}
\hline Describe Your Workload & $\begin{array}{l}\text { Ideal Work } \\
\text { Place }\end{array}$ & $\begin{array}{l}\text { Busy, But } \\
\text { Manageable }\end{array}$ & $\begin{array}{l}\text { Busy, Barely } \\
\text { Manageable }\end{array}$ & Unmanageable \\
\hline Pre-implementation (week 1) & $0 \%$ & $40 \%$ & $40 \%$ & $20 \%$ \\
Pre-implementation (week 2) & $0 \%$ & $50 \%$ & $50 \%$ & $0 \%$ \\
Post-implementation (week 3) & $0 \%$ & $100 \%$ & $0 \%$ & $0 \%$ \\
Post-implementation (week 4) & $0 \%$ & $64 \%$ & $18 \%$ & $18 \%$ \\
Post-implementation (week 5) & $0 \%$ & $100 \%$ & $0 \%$ & $0 \%$ \\
Post-implementation (week 6) & $0 \%$ & $100 \%$ & $0 \%$ & $0 \%$ \\
Post-implementation (week 7) & $20 \%$ & $0 \%$ & $80 \%$ & $0 \%$ \\
\hline
\end{tabular}

\subsection{Staff Experience}

HHSC routinely surveys staff to better understand the staff experience and engagement. Accreditation Canada requires healthcare organizations to use valid, reliable employee engagement tools, such as the Picker Engagement Survey (Lowe 2012). Table 3 displays nurse engagement survey data from the inpatient hematology unit. We will be collecting data for other units in the next round of engagement surveys.

Table 3. HHSC Hematology Nurse Survey Ratings of Engagement.

\begin{tabular}{cccc}
\hline $\begin{array}{c}\text { Staff Engagement Survey Results } \\
\text { (Percentage of Positive Scores) }\end{array}$ & $\begin{array}{c}\text { PRE SYNERGY } \\
\mathbf{N = 3 7}\end{array}$ & $\begin{array}{c}\text { POST SYNERGY } \\
\mathbf{N}=48\end{array}$ & $\begin{array}{c}\text { Chi Square } \\
\text { Test }\end{array}$ \\
\hline Perception of feeling engaged & $43 \%$ & $62 \%$ & $p<0.05$ \\
Perception of the quality of patient care & $62 \%$ & $76 \%$ & $p<0.05$ \\
Perception of workload and work life balance & $24 \%$ & $50 \%$ & $p<0.05$ \\
Stress Satisfaction Offset Scores & $26 \%$ & $56 \%$ & $p<0.05$ \\
\hline
\end{tabular}




\subsection{Nurses' Missed Breaks}

On the hematology unit, the nurses and management helped to select QI indicators. One concern for the nurses was chronic missed breaks. As part of the Study step data in multiple PDSA cycles, approximately 120 nurse shifts were monitored for missed breaks from July 2013 through January 2014. As noted in Figure 4, use of the synergy tool suggests that workloads became more manageable, resulting in decreases in missed breaks.

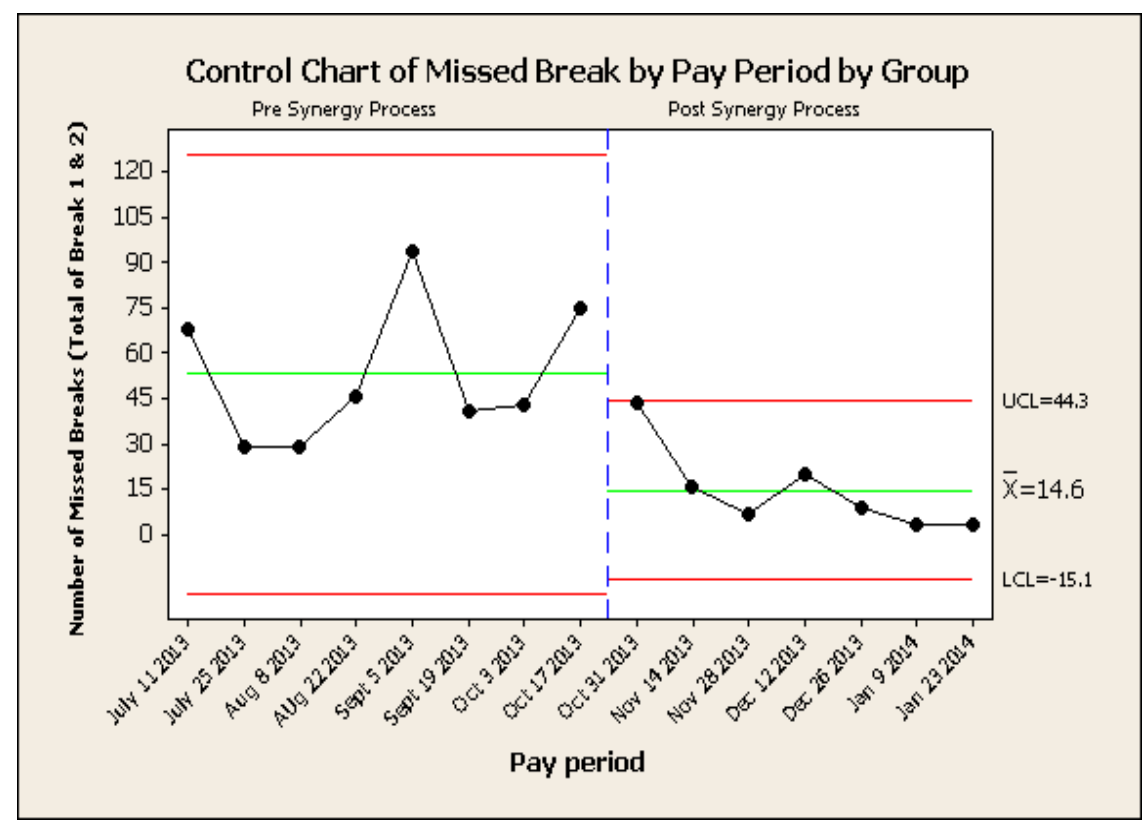

Figure 4. Nurses' Missed Breaks on the HHSC Hematology Unit.

\section{Discussion}

The adaptation of the synergy tool for use across multiple departments at one HHSC acute care site provides a standardized QI approach for identifying patients' priority needs; supports optimal use of nurse competencies; and determines safe, daily staffing assignments for nurses. The use of PDSA cycles promotes a systematic approach to change, with ongoing evidence of improvements in nurses' workloads and levels of engagement. HHSC is now evaluating patient safety outcomes with support from business analytics and quality/safety officers who are part of the organization's QI collaborative. One specific outcome indicator of interest is patient falls with injuries, a major concern with increased numbers of geriatric patients. This indicator is a nurse-sensitive indicator that is associated with nursing workload and safe staffing assignments (Brown et al. 2010).

The initial work of the QI collaborative focused predominantly on nurses' work. Synergy scores are being used by charge nurses and nurse managers to determine the types and numbers of RNs, RPNs, and health care aides needed each shift to provide quality, safe care delivery. We are in the process of examining synergy tool use in an interprofessional context. For example, patients' synergy scores are now included in interprofessional safety rounds and patient case conferences. We are exploring ways to incorporate synergy scores within our electronic health system-for all to use. Currently, patient synergy scores are transferred manually from individual patient management plans to nurse manager worksheets. Once our interprofessional teams have electronic access to synergy scores on an "as needed" basis, we anticipate that we will use patient synergy scores as key decision-making components for admissions and discharge decisions. Our ultimate goal is to ensure that the tool can be used by all healthcare providers across our organization. 


\section{Conclusions}

We began our exploration of the synergy tool as a means to address nurses' workload issues. We recognize that there are many human factors influencing nurses' work at different systems levels. For instance, recent research suggests that workflow interruptions have significant adverse effects on nurse and patient outcomes (Holden et al. 2011; MacPhee et al. 2017). We believe that a successful and empowering way to explore root causes and promote potential workload solutions is by engaging direct care staff throughout the four steps of the PDSA cycle. During our synergy tool pilots, nurses and management co-directed ongoing PDSA cycles, including data collection and interpretation. They provided leadership throughout the staff training and ongoing communications between units and the HHSC QI collaborative. Initial results from our staff engagement surveys support research that demonstrates how participation in QI initiatives enhances engagement and results in greater innovation, organizational commitment, and retention (Bakker et al. 2008).

Acknowledgments: We would like to thank the interprofessional teams on Hematology, Medical-Alternate Level Care and Surgical Oncology units of the Juravinski Hospital and Cancer Center for their daily support and hard work. We also want to thank Georgia Georgiou, Mary Lou Meyers, Gary Blake, Melinda Stacey and Delia Palmar, the managers of these units for their leadership and ongoing commitment to this project. We want to express our gratitude to Kirsten Krull, Vice-President, Quality \& Performance at Hamilton Health Sciences for her vision and guidance in this project.

Author Contributions: E.H., E.P. and C.C. designed the project. Y.A., K.K. and S.H. collected the data and coordinated the project. E.H. provided data analysis. E.H., E.P., C.C. and M.M. interpreted the data. E.H. and E.P. wrote the manuscript. C.C. and M.M. revised the manuscript. All authors read and approved the final manuscript.

Conflicts of Interest: The authors declare no conflict of interest.

\section{Appendix A}

Table A1. Sample timeline using the Plan-Do-Study Act (PDSA) Cycle.

\begin{tabular}{|c|c|}
\hline Activity & Timeline \\
\hline \multicolumn{2}{|l|}{ Plan (Identify Problem, Gather Data, Interpret Data) } \\
\hline Group kick off meeting & Week 1 \\
\hline Revise staff surveys & Week 1 \\
\hline Staff engagement and communication & Week 1-2 \\
\hline \multicolumn{2}{|l|}{ Data collection: baseline data } \\
\hline $\begin{array}{l}\text { - informed consent and administration of survey to staff: (competency assessment, } \\
\text { work environment survey, and assignment evaluation) }\end{array}$ & \multirow{3}{*}{ Week 3} \\
\hline - $\quad$ informed consent and administration of survey to patients & \\
\hline $\begin{array}{l}\text { - Unit specific Safety Occurrence Report, over-time rates, sick time rates, nursing } \\
\text { hours per patient/day }\end{array}$ & \\
\hline Patient characteristic tool development (including face and construct validity) & Week 3 \\
\hline \multicolumn{2}{|l|}{ Do (Act on Evidence) } \\
\hline Training of nurses on patient scoring & Week 5 \\
\hline Pilot patient characteristic tool for 2 weeks & Weeks 6 \& 7 \\
\hline \multicolumn{2}{|l|}{ Study (Evaluate Results) } \\
\hline Analyze data collected from patient characteristic tool & Week 8 \\
\hline Analyze baseline data and correlate with patient characteristic tool & Week 8 \\
\hline Develop staffing guidelines & Week 9 \\
\hline \multicolumn{2}{|l|}{ Act (Identify and Implement Next Steps) } \\
\hline Charge nurse training for patient assignment & Week 9 \\
\hline Identify changes in unit & Week 9 \\
\hline Go live: Implementation of new process & Week 10 \\
\hline \multicolumn{2}{|l|}{ PDSA \#2 (one month post implementation) } \\
\hline Data collection & \\
\hline Analyze and modify tools, improve staffing guidelines & 2 weeks \\
\hline Identify and implement changes in unit & 2 weeks \\
\hline PDSA \#3 (6 months post implementation) & 2 weeks \\
\hline
\end{tabular}




\section{References}

Bakker, Arnold B., Wilmar B. Schaufeli, Michael P. Leiter, and Toon W. Taris. 2008. Work engagement: An emerging concept in occupational health psychology. Work E Stress 22: 187-200.

Berwick, Donald M. 1996. A primer on leading the improvement of systems. BMJ: British Medical Journal 312: 619. [CrossRef] [PubMed]

Brewer, Barbara B., Anne W. Wojner-Alexandrov, Nora Triola, Christine Pacini, Melanie Cline, Jo Ellen Rust, and Karlene Kerfoot. 2007. AACN Synergy Model's characteristics of patients: Psychometric analyses in a tertiary care health system. American Journal of Critical Care 16: 158-67. [PubMed]

Brown, Diane Storer, Nancy Donaldson, Linda Burnes Bolton, and Carolyn E. Aydin. 2010. Nursing-sensitive benchmarks for hospitals to gauge high-reliability performance. Journal for Healthcare Quality 32: 9-17. [CrossRef] [PubMed]

Carter, Kimberly F., and Hilda D. Burnette. 2011. Creating patient-nurse synergy on a medical-surgical unit. MEDSURG Nursing Journal 20: 249-53.

Chambers, John, William Cleveland, Beat Kleiner, and Paul Tukey. 1983. Graphical Methods for Data Analysis. Murray Hill: Wadsworth \& Brooks Publishing Company, pp. 158-62.

Curley, Martha A. Q. 2007. Synergy: The Unique Relationship between Nurses and Patients, the AACN Synergy Model for Patient Care. Indianapolis: Sigma Theta Tau International.

Gralton, Karen S., and Stacy A. Brett. 2012. Integrating the Synergy Model for patient care at Children's Hospital of Wisconsin. Journal of Pediatric Nursing 27: 74-81. [CrossRef] [PubMed]

Holden, Richard J., Matthew C. Scanlon, Neal R. Patel, Rainu Kaushal, Kamisha Escoto, Roger Brown, Samuel J. Alper, Judi M. Arnold, Theresa M. Shalaby, Kathleen Murkowski, and et al. 2011. A human factors framework and study of the effect of nursing workload on patient safety and employee quality of working life. Quality and Safety in Health Care 20: 15-24. [CrossRef] [PubMed]

Khalifehzadeh, Asghar, Mahdi Karimyar Jahromi, and Ahmadreza Yazdannik. 2012. The impact of the Synergy Model on nurses' performance and the satisfaction of patients with acute coronary syndrome. Iranian Journal of Nursing and Midwifery Research 17: 16-20. [PubMed]

Lowe, Graham. 2012. How employee engagement matters for hospital employment. Healthcare Quarterly (Toronto, Ont.) 15: 29-39. [CrossRef]

MacPhee, Maura, Andrea Wardrop, Cheryl Campbell, and Patricia Wejr. 2011. The Synergy professional practice model and its patient characteristics tool: A staff empowerment strategy. Nursing Leadership (Toronto, Ont.) 24: 42-56. [CrossRef]

MacPhee, Maura, V. Susan Dahinten, and Farinaz Havaei. 2017. The impact of heavy perceived nurse workloads on patient and nurse outcomes. Administrative Sciences 7: 7. [CrossRef]

Mainz, Jan. 2003. Defining and classifying clinical indicators for quality improvement. International Journal for Quality in Health Care 15: 523-30. [CrossRef] [PubMed]

Mawhinney, Lynnette. 2010. Let's lunch and learn: Professional knowledge sharing in teachers' lounges and other congregational spaces. Teaching and Teacher Education 26: 972-78. [CrossRef]

Rozdilsky, Janlyn, and Amber Alecxe. 2012. Saskatchewan: Improving patient, nursing and organizational outcomes utilizing formal nurse-patient ratios. Nursing Leadership (Toronto, Ont.) 25: 103-13. [CrossRef]

Schouten, Loes M. T., Marlies E. J. L. Hulscher, Jannes J. E. van Everdingen, Robbert Huijsman, and Richard P. T. M. Grol. 2008. Evidence for the impact of quality improvement collaboratives: Systematic review. BMJ 336: 1491-94. [CrossRef] [PubMed]

Straka, Kristen L., Patricia Brandt, and Jeanne Brytus. 2013. Brief report: Creating a culture of evidence-based practice and nursing research in a pediatric hospital. Journal of Pediatric Nursing 28: 374-78. [CrossRef] [PubMed]

(c) 2017 by the authors. Licensee MDPI, Basel, Switzerland. This article is an open access article distributed under the terms and conditions of the Creative Commons Attribution (CC BY) license (http:// creativecommons.org/licenses/by/4.0/). 